\title{
Huge Mucinous Cystic Neoplasm of the Pancreas in a Young Diabetic Woman
}

\author{
Satoshi Murao ${ }^{1}$, Hitoshi Hosokawa ${ }^{1}$, Kazuaki Yokota ${ }^{2}$, \\ Tetsuji Yamashita $^{2}$ and Takashi Sekikawa ${ }^{2}$
}

Key words: mucinous cystic neoplasm, gestational diabetes, pancreatic diabetes

(Inter Med 47: 2097, 2008)

(DOI: 10.2169/internalmedicine.47.1637)
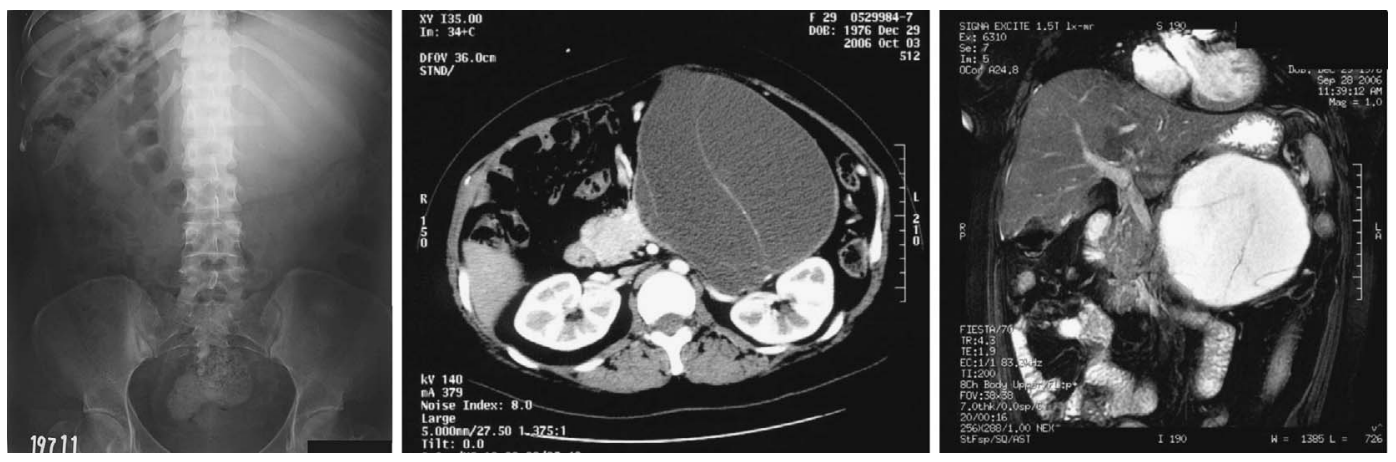

Picture 1. An abdominal mass compressed the intestine (left). A huge cystic mass with septum is observed at the pancreas tail (middle). The cystic mass also compressed the stomach (right). Pancreas body and tail are indistinguishable due to the huge cyst.

A 30-year-old woman who had been diagnosed as gestational diabetes during her first pregnancy at the age of 28 , was referred to our hospital with the complaint of abdominal fullness. Abdominal radiograph, CT, and MRI findings revealed a huge cystic mass of the pancreas compressing other organs (Picture 1). Her height was $145 \mathrm{~cm}$, BW was $70.5 \mathrm{~kg}$, BMI was $33.5 \mathrm{~kg} / \mathrm{m}^{2}$, fasting blood glucose was $155 \mathrm{mg} / \mathrm{dL}$, and HbA1c was $9.4 \%$. Serum levels of immunoreactive insulin and glucagon before/30 minutes after infusion of $30 \mathrm{~g}$ of arginine were $4.72 / 21.1 \mu \mathrm{U} / \mathrm{mL}$ and $32 /$
$270 \mathrm{pg} / \mathrm{mL}$ respectively, indicating decreased insulin secretion without oversecretion of glucagon. Mucinous cystic neoplasm (MCN) was suspected and confirmed histologically. MCN develops with a predilection of pancreas tail in middle aged women. It is usually surgically excised due to its potential of developing malignancy (1). The diabetes of the present case seemed to be pancreatic diabetes. This case also suggests the importance of pancreas imaging at the first diagnosis of diabetes.

\section{Reference}

1. Tanaka M, Chari S, Adsay V, et al. International consensus guidelines for management of intraductal papillary mucinous neoplasms and mucinous cystic neoplasms of the pancreas. Pancreatology 6: 17-32, 2006.

\footnotetext{
${ }^{1}$ Diabetes Center, Takamatsu Hospital, Takamatsu and ${ }^{2}$ Department of Internal Medicine, Matsuyama Shimin Hospital, Matsuyama Received for publication August 27, 2008; Accepted for publication September 1, 2008

Correspondence to Dr. Satoshi Murao, sat.murao@gmail.com

(C) 2008 The Japanese Society of Internal Medicine Journal Website: http://www.naika.or.jp/imindex.html
} 